\title{
COCYCLES OVER ABELIAN TNS ACTIONS
}

\author{
V. NIŢICĂ, A. TÖRÖK
}

\begin{abstract}
We study extensions of higher-rank abelian TNS actions (i.e. hyperbolic and with a special structure of the stable distributions) by compact connected Lie groups. We show that up to a constant, there are only finitely many cohomology classes. We also show the existence of cocycles over higher-rank abelian TNS actions that are not cohomologous to constant cocycles. This is in contrast to earlier results, showing that real valued cocycles, or small Lie group valued cocycles, over higher-rank abelian actions are cohomologous to constants.
\end{abstract}

\section{INTRODUCTION}

The goal of this paper is to describe extensions of abelian totally non-symplectic (abbreviated as TNS; see Definition 2.5) actions by compact Lie groups. These results can be used to classify compact group extensions of certain higher-rank lattice actions on compact manifolds.

This study is motivated by the results obtained recently in the theory of smooth $\mathbb{Z}^{k}$-actions, $k \geq 2$, which show that such actions exhibit strong rigidity properties. In particular, compact Lie group valued cocycles over such actions were expected to be cohomologous to constant cocycles.

We show that this is not exactly the case, but cocycles over abelian TNS actions become (cohomologous to) constant cocycles after possibly taking a finite cover and restricting to a finite index subgroup. Therefore, the cohomology class of a cocycle is described essentially by its values over finitely many points.

This is to be contrasted with the case of cocycles over $\mathbb{Z}$ or $\mathbb{R}$ Anosov actions, where the cohomological classification depends on the values over all periodic orbits, i.e., there are infinitely many independent obstructions. The fact that a cocycle is trivial if its iterates over all periodic orbits are trivial is the content of the celebrated theorem of Livsic ([12], [13]), which applies to $\mathbb{R}$-valued cocycles, and to Lie group valued cocycles that are close to the identity. In [17] one can find an extension of Livsic's results to cocycles with values

Date: Nov. 25, 2002.

Key words and phrases. abelian Anosov actions, totally non-symplectic (TNS), cohomology, equivariant dynamics, principal bundles.

This paper emerged from several discussions that we had with A. Katok and R. Spatzier. We would like to thank them for their interest.

The research of VN was supported in part by NSF Grant DMS 99-71826. The research of AT was supported in part by NSF Grant DMS-0071735. 
in diffeomorphism groups, as well as an extension of all above mentioned results to generic Anosov group actions. The periodic data criterion of Livsic was extended in [19] and [22] to cohomologous cocycles in Lie groups.

Rigidity phenomena appear for actions of larger groups. For lattices in higher-rank Lie groups, this is related to Zimmer's superrigidity theorem [28]. For actions of higher-rank abelian groups $\left(\mathbb{Z}^{k}\right.$ and $\mathbb{R}^{k}, k \geq 2$ ) it was observed by A. Katok and $\mathrm{R}$. Spatzier that cocycle rigidity holds for hyperbolic actions. Using harmonic analysis, they showed in [11] that real valued cocycles over certain Anosov $\mathbb{Z}^{k}$ and $\mathbb{R}^{k}$ actions, $k \geq 2$, are cohomologous to a constant cocycle. A different approach was introduced in [10], based on the notion of a totally non-symplectic $\mathbb{Z}^{k}$ action. It provided a geometric proof of some of the results in [11], and proved the rigidity of certain cocycles taking values in a Lie or diffeomorphism group (see [10] and [18]). For example, it showed that Lie group valued cocycles that are close to the identity are cohomologous to a constant cocycle. Thus, the cohomology class of such cocycles is determined again by a finite amount of data. In [18], diffeomorphism-valued cocycles over abelian TNS actions are used to derive a rigidity result for smooth actions of lattices in higher-rank semisimple Lie groups.

In this paper we consider cocycles that are not necessarily small, and give a complete classification of them. These cocycles take values in a compact connected Lie group. In particular, we find examples of cocycles over TNS actions that are not cohomologous to constant cocycles, in contrast to the above mentioned results. We also show that, up to a constant, there are only finitely many Hölder or smooth cohomology classes for such cocycles. However, it is more convenient to speak about extensions, which are lifts of the action to a principal bundle. Extensions are slightly more general than cocycles, which correspond to lifts to the trivial bundle.

The paper is organized as follows: in $\S 2$ we describe necessary preliminary notions. In $\S 3$ we state a special case of our main results, and give a sketch of the proofs. The general case is presented in $\S 7$. In $\S \S 4$ and 5 we describe how the TNS property implies the existence of an invariant foliation. In $\S 6$ we discuss the holonomies that these foliations can have. The proofs of the main results are given in $\S 7$. In the Appendix we present results about lifting a group action to a principal bundle endowed with a horizontal foliation.

\section{Definitions}

The purpose of this paper is to classify lifts of actions from a manifold $M$ to principal bundles with base $M$.

We recall the definitions of principal bundles and their maps.

Let $M$ be a compact manifold, and $G$ a compact connected Lie group. A $C^{K}, 1 \leq K \leq \omega$, principal $G$-bundle over $M$ consists of a manifold $P$ and an action of $G$ on $M$ satisfying the following conditions:

(i) $G$ acts freely on $P$ on the right: $(\xi, g) \in P \times G \rightarrow \xi g$.

(ii) $M$ is the quotient space of $P$ by the equivalence relation induced by $G, \pi: P \rightarrow M$; 
(iii) $P$ is locally trivial, i.e. every point in $M$ has a neighborhood $U$ such that $\pi^{-1}(U)$ is isomorphic to $U \times G$ in the sense that there is a $C^{K}$-diffeomorphism $\phi: \pi^{-1}(U) \rightarrow$ $U \times G$ such that $\phi(\xi)=(\pi(\xi), \psi(\xi))$, where $\psi: \pi^{-1}(U) \rightarrow G$ satisfies $\psi(\xi g)=\psi(\xi) g$ for all $\xi \in \pi^{-1}(U), g \in G$.

A principal $G$-bundle will be denoted by $P(M, G)$ or simply $P$.

The trivial principal $G$-bundle is $M \times G$, with $G$ acting on itself from the right.

A $G$-map $F: P_{1}\left(M_{1}, G\right) \rightarrow P_{2}\left(M_{2}, G\right)$ between principal $G$-bundles is a continuous mapping that satisfies $F(\xi g)=F(\xi) g$, for all $\xi \in P_{1}, g \in G$. Since a $G$-map takes fibers of $P_{1}$ into fibers of $P_{2}$, it induces a map $f: M_{1} \rightarrow M_{2}$. If $G$ is compact, one can choose Riemannian metrics on $P_{1}, P_{2}$ such that the restriction of $F$ to any fiber becomes an isometry.

Note that if $f: M_{1} \rightarrow M_{2}$ is a homeomorphism, then any $G$-map $F: P_{1} \rightarrow P_{2}$ covering $f$ is a bundle isomorphism. Moreover, any principal $G$-bundle over a smooth manifold is isomorphic to a smooth principal bundle. See $[6, \S 4.3]$. (Similar results are true in the analytic category as well; see [6, $₫ 4.7$, Exercise 3].) Therefore, from now on we will only consider smooth principal bundles.

Definition 2.1. We say that a $G$-map $F: P_{1} \rightarrow P_{2}$ is $\theta$-Hölder (with respect to a specified metric on the bundles), respectively $C^{K}$, if it is given by $\theta$-Hölder, respectively $C^{K}$, maps in a family of local trivializations of the bundles.

Definition 2.2. Consider an action of a (discrete) group $\mathcal{A}$ on the manifold $M, \alpha: \mathcal{A} \times M \rightarrow$ $M$. A $G$-extension of $\alpha$ is a principal $G$-bundle $p: P \rightarrow M$ endowed with a lift of $\alpha$ to an action $\widetilde{\alpha}: \mathcal{A} \times P \rightarrow P$ through $G$-maps.

In the special case when $P$ is the trivial bundle $M \times G$, an extension $\widetilde{\alpha}$ of $\alpha$ is described by a cocycle $\beta: \mathcal{A} \times M \rightarrow G$ :

$$
\widetilde{\alpha}_{a}(x, g)=(\alpha(x), \beta(a, x) g), \quad x \in M, g \in G, a \in \mathcal{A} .
$$

Recall that $\widetilde{\alpha}$ is an action if and only if $\beta$ satisfies the cocycle equation

$$
\beta(a b, x)=\beta(a, b(x)) \beta(b, x), \quad a, b \in \mathcal{A}, x \in M .
$$

A cocycle is constant if it does not depend on $x \in M$. In that case, $\beta(a, x)=\rho(a)$, where $\rho: \mathcal{A} \rightarrow G$ is a group homomorphism.

We now describe the natural equivalence relation between extensions, which corresponds to the cohomology of cocycles.

Definition 2.3. Two $G$-extensions $\widetilde{\alpha}_{i}: \mathcal{A} \times P_{i} \rightarrow P_{i}, i=1,2$, of $\alpha$ are cohomologous if there is a $G$-bundle isomorphism between $\widetilde{\alpha}_{1}$ and $\widetilde{\alpha}_{2}$, i.e., a $G$-bundle map $F: P_{1} \rightarrow P_{2}$ covering Id $: M \rightarrow M$ such that

$$
\widetilde{\alpha}_{2}(a)=F \circ \widetilde{\alpha}_{1}(a) \circ F^{-1} \quad \text { for each } a \in \mathcal{A} .
$$

We call $F$ the transfer function. 
Note that if two $G$-extensions are cohomologous through a continuous transfer map, then the corresponding principal bundles are isomorphic in the $C^{K}$-category as well, $1 \leq K \leq \infty$ $[6, \S 4.3]$. A similar conclusion in the analytic category follows from [6, $\S \S 4.3$ and 4.7] and [26]; the result of Tognoli [26] applies to infranilmanifolds (described below), which are homogeneous spaces. Hence, in our case it is enough to consider the cohomology question only for extensions on the same bundle.

For extensions on the trivial bundle $M \times G$, the cohomology equation (2.2) becomes

$$
\beta_{2}(a, x)=f(a(x)) \beta_{1}(a, x) f(x)^{-1},
$$

where $F(x, g)=(x, f(x) g)$.

We will be interested only in extensions of TNS actions of $\mathcal{A}=\mathbb{Z}^{k}, k \geq 2$, which are a particular case of hyperbolic actions. We first recall the definition of a partially hyperbolic diffeomorphism, and then define TNS actions.

Let $M$ be a compact manifold. A $C^{1}$-diffeomorphism $T: M \rightarrow M$ is called partially hyperbolic if there is a continuous invariant splitting of the tangent bundle $T M=E^{s}(T) \oplus$ $E^{0}(T) \oplus E^{u}(T)$ such that the derivative $T f$ expands $E^{u}(T)$ much sharper than $E^{0}(T)$, and contracts $E^{s}(T)$ much sharper than $E^{0}(T)$. See [7] and [2] for the theory of partially hyperbolic diffeomorphisms, as needed for this paper. The sub-bundles $E^{s}(T)$ and $E^{u}(T)$ are called the stable, respectively unstable, distributions. These distributions are integrable. We denote by $W^{s}(x ; T)$ and $W^{u}(x ; T)$ the stable, respectively unstable manifolds of the point $x \in M$. The stable and unstable foliations are Hölder foliations. If the diffeomorphism $T$ is in $C^{K}(M)$, then the leaves of the stable and unstable foliations are $C^{K}$ too. Recall that the center distribution $E^{0}(T)$ is not always integrable.

If $E^{0}=\{0\}$ then the diffeomorphism $T$ is called Anosov. The only manifolds which are known to admit Anosov diffeomorphisms are tori, nilmanifolds and infranilmanifolds. It is an outstanding conjecture that these are the only ones supporting Anosov diffeomorphisms (see [4]).

A nilmanifold is the quotient of a connected, simply connected nilpotent Lie group $N$ by a lattice $\Gamma$. All such lattices are cocompact, torsion free and finitely generated; see [21], Theorems 2.1 and 2.18. An example of nilmanifold is $\mathbb{T}^{d}$, the $d$-dimensional torus. An infranilmanifold is finitely covered by a nilmanifold. More precisely, let $N$ be a connected, simply connected nilpotent Lie group and $C$ a compact group of automorphisms of $N$. Let $\Gamma$ be a torsion free cocompact discrete subgroup of the semi-direct product $N C$. Recall that an element $(x, c)$ of $N C$ (where $x \in N$ and $c \in C$ ) acts on $N$ by first applying $c$ and then left translating by $x$. By a result of Auslander (see [1]), $\Gamma \cap N$ is a cocompact discrete subgroup of $N$ and $\Gamma \cap N$ has finite index in $\Gamma$. The quotient space $N / \Gamma \cap N$ is a compact manifold called an infranilmanifold.

Anosov diffeomorphisms on nilmanifolds and infranilmanifolds were introduced in [24], [4] and [23]. Let $\bar{f}: N C \rightarrow N C$ be an automorphism for which $\bar{f}(\Gamma)=\Gamma, \bar{f}(N)=N$. Then $\bar{f}$ induces a diffeomorphism $f: N / \Gamma \cap N \rightarrow N / \Gamma \cap N$, called an infranilmanifold automorphism. If the derivative $\left.D \bar{f}\right|_{N}$ at the identity is hyperbolic, i.e. has all eigenvalues of absolute value 
different from 1, then $f$ is an Anosov diffeomorphism. Note that in this case the stable and unstable distributions are smooth.

In this paper we consider $\mathbb{Z}^{k}$-actions only on infranilmanifolds.

Definition 2.4. We call an action linear if it is given by infranilmanifold automorphisms.

Recall the Franks-Manning classification of Anosov diffeomorphisms on infranilmanifolds (see [3], [14] for the case of a $\mathbb{Z}$-action and [8], proof of Proposition 2.18, for the case of a $\mathbb{Z}^{k}$-action). Let $M$ be an infranilmanifold and $\alpha: \mathbb{Z}^{k} \times M \rightarrow M$ an abelian $C^{1}$ action containing an Anosov diffeomorphism. Assume that $\alpha$ has a fixed point $x_{0}$. Then the action $\alpha$ is Hölder conjugate to the linear $\mathbb{Z}^{k}$-action $\bar{\alpha}: \mathbb{Z}^{k} \times M \rightarrow M$ given by automorphisms induced by the map in homotopy $\alpha_{*}: \mathbb{Z}^{k} \times \pi_{1}\left(M, x_{0}\right) \rightarrow \pi_{1}\left(M, x_{0}\right)$. Note that the action $\alpha$ always has a periodic point. In general the action $\alpha$ is Hölder conjugate to an affine action, whose restriction to a subgroup of $\mathbb{Z}^{k}$ of finite index is an action by linear automorphisms.

Let $\alpha: \mathbb{Z}^{k} \times M \rightarrow M$ be an abelian $C^{K}$ action. View $\alpha$ as a homomorphism from $\mathbb{Z}^{k}$ into $\operatorname{Diff}^{K}(M)$ and denote by $\mathcal{A} \subset \operatorname{Diff}^{K}(M)$ its image.

TNS actions were introduced in [10]:

Definition 2.5. We say that an action $\alpha$ is totally non-symplectic, or TNS, if there is a family $S$ of partially hyperbolic elements in $\mathcal{A}$ and a continuous splitting of the tangent bundle $T M=\oplus_{i=1}^{m} E_{i}$ into $\mathcal{A}$-invariant distributions such that:

(i) the stable and unstable distributions of any element in $S$ are direct sums of a subfamily of the $E_{i}$ 's;

(ii) any two distributions $E_{i}$ and $E_{j}, 1 \leq i, j \leq m$, are included in the stable distribution of some element in $S$.

If, moreover, the action $\alpha$ is $C^{\infty}$ and each distribution $E_{i}$ is smooth, we say that the action is smoothly-TNS.

It is easy to see that we can assume that the set $S$ consists only of Anosov elements, and that the distributions $E_{i}$ are integrable (see [10, $§ 2$ Remarks]).

If $G$ is compact and the extension is at least $C^{1}$, it follows from the discussion below that the lift of any Anosov element in $S$ is a partially hyperbolic diffeomorphism. For such a map, the center distribution is integrable and the center leaves are given by the orbits of the $G$-action. In addition, the local stable and unstable leaves of the lift are graphs of $G$-valued functions defined over the local stable and unstable leaves in the base. For Hölder extensions a similar result is true (see Proposition 4.1).

\section{The MAin Results (A SPECIAL CASE)}

Our results state that cohomology classes of cocycles over (respectively, extensions of) TNS actions are essentially given by constant cocycles. Modulo the choice of the constant, there are only finitely many possibilities. This is an extension of the results in [10] where it was proven that small Lie group valued cocycles are cohomologous to a constant. 
To be precise, assume that $M$, the principal bundles and the action $\alpha$ are smooth (meaning analytic, $C^{\infty}$, or a sufficiently large $C^{L}$ ). The extensions, cocycles and transfer maps can be measurable, $\theta$-Hölder or $C^{K}, 1 \leq K \leq \omega$. The smoothness of the transfer map $F$ determines the type of cohomology: if $F$ is continuous we speak about continuous cohomology classes, if it is smooth of smooth cohomology classes, etc.

Note that by the results of [5] (see also [13]), if two $\theta$-Hölder extensions on the same bundle are cohomologous through a measurable transfer function $F$, then $F$ coincides a.e. with a $\theta$-Hölder function. From [16], it follows that if two $C^{K}$-extensions are cohomologous through a continuous transfer function $F$, then $F$ is $C^{K-\varepsilon}$. Here $K-\varepsilon=K$ for $K \in\{1, \infty, \omega\}$; for $K=\omega$ one needs that the bundles be also $C^{\omega}$-isomorphic, which in many cases follows from their continuous isomorphism (see [6, $\S \S 4.3$ and 4.7] and [26]).

For brevity, we are going to discuss our results only for the case of the continuous cohomology. The smooth and measurable counterparts follow from the results mentioned above.

We state first our results for the case when the action on the base $M$ has a fixed point. The general case, i.e., when there are only periodic points (those having a finite $\mathcal{A}$-orbit), is considered in $\S 7$. Given a map $a: M \rightarrow M$, we denote by $a_{*}$ its action on homotopy.

Theorem 3.1. Assume $M$ is an infranilmanifold and the smoothly-TNS action $\alpha: \mathcal{A} \times M \rightarrow$ $M$ has a fixed point $x_{0}$.

(a) The continuous cohomology classes of $\mathbb{T}^{\kappa}$-valued smooth cocycles are in one-to-one correspondence with pairs of homomorphisms

$$
\bar{H}: V \rightarrow \mathbb{T}^{\kappa}, \quad \beta: \mathcal{A} \rightarrow \mathbb{T}^{\kappa},
$$

where

$$
V:=\pi_{1}\left(M, x_{0}\right) / \operatorname{span}\left\{a_{*}(\omega) \omega^{-1} \mid \omega \in \pi_{1}\left(M, x_{0}\right), a \in \mathcal{A}\right\}
$$

is a finite group (determined completely by the action).

In particular, there are TNS actions that admit smooth cocycles not cohomologous to a constant. See Proposition 3.4.

(b) Let $G$ be a compact connected Lie group. The continuous cohomology classes of smooth $G$-extensions of $\alpha$ are in one-to-one correspondence with conjugacy classes of pairs of homomorphisms $(H, \beta)$, where

$$
H: \pi_{1}\left(M, x_{0}\right) \rightarrow G, \quad \beta: \mathcal{A} \rightarrow G,
$$

and $H$ intertwines the actions $\alpha_{*}: \mathcal{A} \rightarrow \operatorname{Aut}\left(\pi_{1}\left(M, x_{0}\right)\right)$ and $\beta_{*}:=\operatorname{Int} \circ \beta: \mathcal{A} \rightarrow$ $\operatorname{Aut}(G)$. That is,

$\left.H\left(\alpha_{*}(a)(\omega)\right)=\beta_{*}(a)(H(\omega))\right)=\beta(a) H(\omega) \beta(a)^{-1}, \quad a \in \mathcal{A}, \omega \in \pi_{1}\left(M, x_{0}\right)$.

Up to conjugacy, there are only finitely many homomorphisms $H$, and each has a finite image. Therefore, each extension has a finite cover which is a trivial G-bundle and on which the lifted $\mathcal{A}$-action is cohomologous to a constant cocycle. This cover can be chosen to be the same for all $G$-extensions of $\alpha$. 
Remark. We say that two pairs of maps $(H, \beta)$ and $\left(H^{\prime}, \beta^{\prime}\right)$ into $G$ are conjugated if there is an element $g \in G$ such that $H^{\prime}=\operatorname{Int}_{g} \circ H$ and $\beta^{\prime}=\operatorname{Int}_{g} \circ \beta$, where $\operatorname{Int}_{g}(h)=g h g^{-1}$.

If the action is on a torus, we can classify Hölder extensions over arbitrary TNS actions:

Theorem 3.2. Assume $M \cong \mathbb{T}^{d}$ and the TNS action $\alpha: \mathcal{A} \times M \rightarrow M$ has a fixed point $x_{0}$.

Then the conclusions of Theorem 3.1 hold for continuous cohomology classes of $\theta$-Hölder $\mathbb{T}^{\kappa}$-cocycles, respectively continuous cohomology classes of $\theta$-Hölder G-extensions.

In particular, any Hölder cocycle (respectively extension) is Hölder cohomologous to a smooth one.

The proofs of both theorems are given in $\S 7$.

Remark. Since there are only finitely many bundles that admit extensions, one needs a finite amount of information to determine on which bundle a given extension is defined. For example, assume $\alpha$ is a TNS action on the infranilmanifold $M$ and $G$ is a connected compact Lie group. Then there is a neighborhood $U$ of the identity in $G$ and finitely many images of the holonomy map, which can be computed explicitly using trivializations of the bundle over a fixed finite set of periodic points, such that a $G$-extension $\widetilde{\alpha}$ acts on a trivial bundle and is cohomologous to a product action $\alpha \times \beta$ if and only if the images of the holonomy map are in $U$.

Next, we describe a family of TNS actions which we use to construct cocycles not cohomologous to a constant.

Example 3.3. Let $\mathrm{SL}(n, \mathbb{R})$ be the group of real-valued invertible matrices of determinant one. Let $\mathbf{T} \subset \operatorname{SL}(n, \mathbb{R})$ be a maximal torus such that $\mathbf{T} \cong \mathbb{R}^{n-1}$. Let $\operatorname{SL}(n, \mathbb{Z}) \subset \operatorname{SL}(n, \mathbb{R})$ be the subgroup with integer entries, and let $\Gamma$ be a subgroup of finite index in $\operatorname{SL}(n, \mathbb{Z})$. Then $\Gamma$ is a lattice in $\operatorname{SL}(n, \mathbb{R})$, and it follows from a theorem of Prasad-Raghunathan (see [20], Theorem 7.1), that there is $g \in \operatorname{SL}(n, \mathbb{R})$ such that $\mathcal{A}:=g \mathbf{T} g^{-1} \cap \Gamma$ is a cocompact lattice in $\mathbf{T}$. In particular, it follows that:

(1) $\mathcal{A}-\{I\}$ consists of hyperbolic matrices;

(2) The elements of $\mathcal{A}$ are simultaneously diagonalizable over $\mathbb{R}$;

(3) $\mathcal{A}$ is isomorphic to a free abelian group of rank $n-1$;

(4) If $v_{1}, \ldots, v_{n} \in \mathbb{R}^{n}$ is a basis of simultaneous eigenvectors for the group $\mathcal{A}$, and $\lambda_{i}$ : $\mathcal{A} \rightarrow \mathbb{R}^{n}$ is the character of $\mathcal{A}$ defined via $A v:=\lambda_{i}(A) v_{i}, A \in \mathcal{A}$, then for any strict nonempty subset $J$ of $\{1, \ldots, n\}$, there exists $A \in \mathcal{A}$, such that $\lambda_{j}(A)<1$, for $j \in J$, and $\lambda_{i}(A)>1$, for $i \notin J$;

Using property 4 above, it follows that the natural action of $\mathcal{A}$ on $\mathbb{T}^{n}$ is a TNS action of $\mathbb{Z}^{n-1}$

Proposition 3.4. Let $n \geq 3, \kappa \geq 1$. There are TNS actions $\alpha: \mathcal{A} \times \mathbb{T}^{n} \rightarrow \mathbb{T}^{n}$ that have a fixed point $x_{0}$, and nontrivial homomorphisms $\bar{H}: V \rightarrow \mathbb{T}^{\kappa}$, where

$$
V:=\pi_{1}\left(\mathbb{T}^{n}, x_{0}\right) / \operatorname{span}\left\{a_{*}(\omega) \omega^{-1} \mid \omega \in \pi_{1}\left(\mathbb{T}^{n}, x_{0}\right), a \in \mathcal{A}\right\}
$$


In particular, any cocycle in a cohomology class corresponding to such a homomorphism is not cohomologous to a constant.

Proof. Let $k \geq 2$ be an integer. Let $\Gamma \subset \operatorname{SL}(n, \mathbb{Z})$ be the congruence subgroup of order $k$, i.e. $\Gamma=\{A \in \operatorname{SL}(n, \mathbb{Z}) \mid A \cong \operatorname{Id}(\bmod k)\}$. Then $\Gamma$ has finite index in $\operatorname{SL}(n, \mathbb{Z})$ and we obtain a TNS action of an abelian group $\mathcal{A} \cong \mathbb{Z}^{n-1}$ by the method described in Example 3.3. The subgroup $\operatorname{span}\left\{a_{*}(\omega) \omega^{-1} \mid \omega \in \pi_{1}\left(\mathbb{T}^{n}, x_{0}\right), a \in \mathcal{A}\right\}$ is included in $k \pi_{1}\left(\mathbb{T}^{n}, x_{0}\right)$ (note that $\pi_{1}\left(\mathbb{T}^{n}, x_{0}\right) \cong \mathbb{Z}^{n}$ is abelian). Hence $V$ is a non-trivial finite abelian group. Any such group is a direct product of cyclic finite groups. If $\mathbb{Z}_{p}$ is a non-trivial factor of order $p$, one can map the generator of $\mathbb{Z}_{p}$ into an element of order $p$ in $\mathbb{T}^{\kappa}$ and find a non-trivial homomorphism $\bar{H}$.

An important ingredient of our proofs is the existence of an invariant foliation on a principal bundle. Namely:

Definition 3.5. Let $P$ be a principal $G$-bundle over $M$. A horizontal foliation $\mathcal{F}$ of $P$ is a continuous $G$-invariant foliation such that each leaf of $\mathcal{F}$ covers $M$ and is transverse to the fibers of $P$. That is, locally the leaves are $G$-translates of the images of continuous sections $\widetilde{\gamma}_{x}: V_{x} \rightarrow P$, where $V_{x}$ is a neighborhood of $x$.

We call the foliation $\mathcal{F}$ Hölder or $C^{K}$ if its leaves are Hölder or $C^{K}$ (that is, the sections $\widetilde{\gamma}_{x}$ describing them are Hölder or $C^{K}$ ).

Note that having a smooth horizontal foliation on the principal $G$-bundle $P$ is identical to specifying a $G$-invariant flat connection of $P$.

We outline now the method. As seen in $\S 2$, the lifted action $\widetilde{\alpha}$ on $P$ is partially hyperbolic. We show in $\S \S 4$ and 5 that — due to the TNS property of $\alpha$ - the strong stable and strong unstable foliations "commute" locally. Thus $W^{u}$ and $W^{s}$ can be integrated into an $\widetilde{\alpha}$-invariant horizontal foliation $\mathcal{F}$ of $P$.

The map $H: \pi_{1}\left(M, x_{0}\right) \rightarrow G$ is the holonomy of this foliation, and $\bar{H}$ is the homomorphism it induces to $V$. The homomorphism $\beta$ is the restriction of $\widetilde{\alpha}$ to the fiber over the fixed point $x_{0}$. [If there are no fixed points, one has to take into account the action of $\widetilde{\alpha}$ over the $\alpha$-orbit of a periodic point.]

The invariance properties of $\mathcal{F}$ are equivalent to the condition (3.1). As shown in [10], if both $H$ and $\beta$ are close to $e_{G}$ on a set of generators, these equations admit only the trivial solution $H \equiv e_{G}$. We prove in $\S 6$ that $H$ always has a finite image, and, up to conjugacy, there are only finitely many choices.

Since a principal $\mathbb{T}^{\kappa}$-bundle that admits a horizontal foliation is isomorphic to the trivial bundle $M \times \mathbb{T}^{\kappa}$, all $\mathbb{T}^{\kappa}$-extensions can be realized by cocycles. This is expressed in the first part of the theorems.

\section{Foliations in the Hölder CASE}

Recall that we assumed that the principal bundles and the action on the base $M$ are smooth; the extensions to the principal bundles may be only Hölder. 
In this section we prove:

Proposition 4.1. Assume that $\mathcal{A} \subset \operatorname{Diff}\left(\mathbb{T}^{n}\right)$ is a TNS action, $G$ is a compact connected Lie group, and $P \rightarrow M$ is a principal $G$-bundle that admits a Hölder $G$-extension $\widetilde{\alpha}: \mathcal{A} \times P \rightarrow P$ of $\alpha$.

Then there exists an $\widetilde{\alpha}$-invariant Hölder horizontal foliation $\widetilde{W}$ of P. Moreover, this is the unique continuous $\widetilde{\alpha}$-invariant horizontal foliation of $P$.

Remark. If the group $G$ is not compact, one can prove similar results for extensions that, in corresponding coordinate charts, are given by cocycles close to the identity. But since this might force the bundle to be trivial, in which case the results of [10] apply, we will not discuss this here.

The proof of Proposition 4.1 follows as in [10], once we extend some of the results to the case of principal bundles. We explain this below.

The main consequence of the TNS property is the fact that the stable and unstable foliations of the $G$-extensions commute. The existence of stable foliations for Hölder extensions is proven in Lemma 4.2, and the property we need in Lemma 4.3.

These two Lemmas establish the basic facts on which [10, $\S \S 4$ and 5] rely. Indeed, Lemma 4.2 takes care of [10, Lemma 4.1]. The conclusions (i) and (ii) of [10, Lemma 4.2] are provided by Lemma 4.3, while (iii) is given by equation (4.3).

The construction of the invariant foliation is done locally, using the results proved so far (in [10]: see the description of the foliation after Lemma 4.2, which relies on formula (4.5); this formula is proven in Lemmas 5.1-5.4; invariance and regularity are proven in Lemma 4.3). Therefore, it does not matter whether the principal bundle is trivial (as in [10]) or not.

We first state the two Lemmas for principal bundles, and then give their proofs.

Lemma 4.2. Let $f: M \rightarrow M$ be an Anosov diffeomorphism, and $p: P \rightarrow M$ a principal $G$-bundle. Let $W=\{W(x)\}_{x \in M}$ be an $f$-invariant foliation of $M$ whose leaves are included in the stable foliation of $f$. Assume that $F$ is a $\theta$-Hölder $G$-extension of $f$ to $P$.

Then there exists an $F$ - and $G$-invariant continuous foliation $\widetilde{W}=\{\widetilde{W}(\xi)\}_{\xi \in P}$ of $P$ whose leaves are lifts of the leaves of $W$ (i.e., locally, the leaves of $\widetilde{W}$ are obtained by G-translates of graphs of continuous sections $\widetilde{\gamma}_{x}^{W}: W_{\text {loc }}(x) \rightarrow P$, which properly normalized, vary in the uniform metric continuously with respect to $x)$. Moreover, this is the unique foliation with these properties, and the sections $\widetilde{\gamma}_{x}^{W}$ are actually uniformly $\theta$-Hölder on $W_{\text {loc }}(x)$.

If $W$ and $F$ are smooth, then $\widetilde{\gamma}_{x}^{W}$ are uniformly smooth on $W_{\mathrm{loc}}(x)$ (i.e. $\widetilde{W}$ has smooth leaves).

Remark. If we apply the above Lemma to $W=W_{f}^{s}$, the stable foliation of $f$, then the foliation $\widetilde{W}$ is the "stable foliation" of $F$ :

$$
\eta \in W^{s}(\xi ; F) \Longleftrightarrow \lim _{n \rightarrow \infty} \operatorname{dist}\left(F^{n}(\eta), F^{n}(\xi)\right)=0 .
$$

Denote the corresponding local sections $\widetilde{\gamma}_{x}^{W_{f}^{s}}$ by $\widetilde{\gamma}_{x}^{F}$. 
If $W \subset W_{f}^{s}$, then $\widetilde{\gamma}_{x}^{W}$ are the restrictions of $\widetilde{\gamma}_{x}^{F}$ to $W$. Hence, except for the uniqueness part, it is enough to prove Lemma 4.2 for $W=W_{f}^{s}$.

Lemma 4.3. Let $\alpha: \mathcal{A} \times M \rightarrow M$ be a group acting on $M$ which has a Hölder extension $\widetilde{\alpha}: \mathcal{A} \times P \rightarrow P$ to the principal $G$-bundle $P \rightarrow M$. Assume that $a, b \in \mathcal{A}$ commute and $\alpha(a), \alpha(b)$ are hyperbolic diffeomorphisms. Denote by $\widetilde{a}$ and $\widetilde{b}$ their extension to $P$.

Then $\widetilde{a}$ preserves the stable foliation $W_{\widetilde{b}}^{s}$ and therefore, up to right translation by a group element,

$$
\left.\widetilde{\gamma}_{x}^{\widetilde{a}}\right|_{W_{\mathrm{loc}}^{s}(x ; \alpha(a)) \cap W_{\mathrm{loc}}^{s}(x ; \alpha(b))}=\left.\widetilde{\gamma}_{x}^{\widetilde{b}}\right|_{W_{\mathrm{loc}}^{s}(x ; \alpha(a)) \cap W_{\mathrm{loc}}^{s}(x ; \alpha(b))} .
$$

Remark. Note that formula (4.2) is property (i) of [10, Lemma 4.2]. The fact that $\widetilde{a}$ preserves the stable foliation of $\widetilde{b}$ is exactly property (ii). Property (iii) expresses the fact that $W_{F}^{s}$ is indeed a foliation, which is an immediate consequence of equation (4.1):

$$
\eta \in W^{s}(\xi ; F), \xi \in W^{s}(\zeta ; F) \Longrightarrow \eta \in W^{s}(\zeta ; F) \text {. }
$$

\section{Proof of Lemma 4.2 .}

Assuming that $W, F$, and the stable foliation of $f$ are smooth, the last statement of the Lemma follows from the general theory of stable foliations of partially hyperbolic diffeomorphisms. If the stable foliation of $f$ is not smooth, we can use the same techniques, or the formula of $\widetilde{\gamma}_{x}^{W}$ obtained below for the Hölder case and the explicit computations of [16, Appendix], to reach the desired conclusion. Thus, we only need to prove the Hölder case.

By the Remark following Lemma 4.2, it is enough to prove the existence and regularity of $\widetilde{\gamma}$ for $W=W_{f}^{s}$, and check uniqueness for a general $W$.

We adapt the proof of Lemma 4.1 in [10] to the case of principal bundles. The estimates are the same, except that we have to deal with a family of Hölder maps instead of a single one.

Consider a family of $G$-bundle charts $\left\{\phi_{i}: p^{-1}\left(U_{i}\right) \subset P \rightarrow U_{i} \times G\right\}_{i \in \mathcal{I}}$, where $\left\{U_{i}\right\}_{i \in \mathcal{I}}$ is a finite open cover of $M$. Hence $\phi_{i}(\xi g)=\phi_{i}(\xi) g$ for $\xi \in p^{-1}\left(U_{i}\right)$ and $g \in G$.

Let $r^{\prime}>0$ be a Lebesgue constant of the covering $\left\{U_{i}\right\}_{i \in \mathcal{I}}$ (for each $x$, the open ball $B_{r^{\prime}}(x)$ of diameter $r^{\prime}$ is contained in one of the $U_{i}$ 's).

Choose a "domain" function $d: M \rightarrow \mathcal{I}$ such that $B_{r^{\prime}}(x) \subset U_{d(x)}$.

We will sometimes use the notation $\phi_{x}$ for $\phi_{d(x)}$, respectively $U_{x}$ for $U_{d(x)}$.

For $x \in M$ let us denote $x_{n}:=f^{n}(x)$.

Since $f$ is uniformly continuous, there is a constant $r>0$ such that $f\left(B_{r}(x)\right) \subset B_{r^{\prime}}(f(x))$ for each $x \in M$.

Given $x \in M, n \in \mathbb{Z}$, and $i, j \in \mathcal{I}$ such that $x \in U_{i}, f^{n}(x) \in U_{j}$, let us denote by $\beta_{j, i}(n, x) \in G$ the "fiber component" of $F^{n}: p^{-1}(x) \rightarrow p^{-1}\left(f^{n}(x)\right)$ in the charts $\phi_{i}$ and $\phi_{j}$, that is

$$
F^{n}\left(\phi_{i}^{-1}(x, g)\right)=\phi_{j}^{-1}\left(f^{n}(x), \beta_{j, i}(n, x) g\right), \quad g \in G .
$$

Therefore $\beta_{k, j}\left(n, f^{m}(x)\right) \beta_{j, i}(m, x)=\beta_{k, i}(n+m, x)$. 
If $i=d(x)$ and $j=d\left(f^{n}(x)\right)$ then we write $\beta(n, x)$ for $\beta_{j, i}(n, x)$. We will also use $\beta_{x, y}$ for $\beta_{d(x), d(y)}$.

With these notations, we claim that the stable manifolds of $F$ are given by the images of

$$
t \in W^{s}(x ; f) \subset M \mapsto \widetilde{\gamma}_{x}^{F}(t):=\phi_{t}^{-1}\left(t, \gamma_{x}^{F}(t) g\right) \in P
$$

where $g \in G$ and

$$
\gamma_{x}^{F}(t)=\lim _{n \rightarrow \infty}\left[\beta_{x_{n}, t}(n, t)\right]^{-1} \beta(n, x) .
$$

The expression in (4.6) is considered from the domain of the chart $\phi_{x}$ to the domain of the chart $\phi_{t}$. Note that for $n$ large, $f^{n}(t) \in U_{d\left(f^{n}(x)\right)}$.

Indeed, once the convergence in (4.6) is proven, one can check that if $t \in W^{s}(x ; f), n$ is large enough, $\xi=\phi_{x}^{-1}(x, g)$ and $\eta=\phi_{t}^{-1}\left(t, \gamma_{x}(t) g\right)$, then

$$
\begin{gathered}
\phi_{x_{n}}\left(F^{n}(\xi)\right)=\left(x_{n}, \beta(n, x) g\right), \\
\phi_{x_{n}}\left(F^{n}(\eta)\right)=\left(t_{n}, \beta_{x_{n}, t}(n, t) \gamma_{x}(t) g\right),
\end{gathered}
$$

and, using the biinvariance of the metric on $G$,

$$
\lim _{n \rightarrow \infty} \operatorname{dist}_{G}\left(\beta(n, x) g, \beta_{x_{n}, t}(n, t) \gamma_{x}(t) g\right)=0,
$$

which shows that $\eta \in W^{s}(\xi ; F)$.

Next we prove that the limit in (4.6) exists, and that the functions $\widetilde{\gamma}_{x}^{F}$ are Hölder and describe a continuous $F$-invariant foliation.

Define, for $t \in W^{s}(x ; f)$ and $n$ so large that $t_{n} \in U_{x_{n}}$,

$$
\gamma_{n, x}(t)=\beta_{x_{n}, t}(n, t)^{-1} \beta(n, x)
$$

(we ignore from now on the superscript $F$ of $\gamma$ ). Then

$$
\gamma_{n, x_{k}}\left(t_{k}\right)=\beta(k, t) \gamma_{n+k, x}(t) \beta(k, x)^{-1},
$$

which shows that it is enough to prove (4.6) for $t$ close to $x$ (i.e., $k$ large), and that the limit satisfies the desired $F$-invariance. But then $t_{n} \in B_{r}\left(x_{n}\right)$ for all $n \geq 0$ and therefore one can write both $\beta(n, x)$ and $\beta_{x_{n}, x}(t)$ as products of the cocycles $\beta_{k}:=\beta_{x_{k+1}, x_{k}}(1, \cdot), k \geq 0$, defined on $B_{r}\left(x_{k}\right)$. Since there are only finitely many charts, these cocycles have a uniformly bounded Hölder norm, independently of $x$.

Therefore, repeating the computations of [10, Lemma 4.1] for the products of $\beta_{k}$ (instead of the same $\beta$ ) shows that

$$
\gamma_{x}^{\prime}(t):=\lim _{n \rightarrow \infty} \beta_{x_{n}, x}(n, t)^{-1} \beta(n, x)
$$

has the desired properties: the limit exists and is uniformly Hölder on $W_{\text {loc }}^{s}(x ; f)$. Since for $t$ close to $x$,

$$
\widetilde{\gamma}_{x}^{F}(t)=\phi_{x}^{-1}\left(t, \gamma_{x}^{\prime}(t)\right),
$$

we obtain the desired result. 
We now prove the uniqueness part of the Lemma. Assume that $\widetilde{\omega}_{x}: W_{\text {loc }}(x) \rightarrow P$ is a family of continuous sections whose $G$-translates give an $F$-invariant continuous foliation. We can "normalize" these sections by a translation, so that $\phi_{x}\left(\widetilde{\omega}_{x}(x)\right)=(x, \mathrm{Id})$.

The $F$-invariance implies that

$$
F^{n}\left(\widetilde{\omega}_{x}(t)\right)=\widetilde{\omega}_{f^{n}(x)}\left(f^{n}(t)\right) c_{n, x}
$$

where $c_{n, x} \in G$ does not depend on $t \in W(x)$. Computing at $t=x$ in the charts $\phi_{x}$ and $\phi_{f^{n}(x)}$, one finds that $c_{n, x}=\beta(n, x)$. Note that the same conclusion applies for $\widetilde{\gamma}_{x}$ as well (formulas (4.5) and (4.6) show that $\phi_{x}\left(\widetilde{\gamma}_{x}(x)\right)=(x$, Id) too).

Since both $\widetilde{\omega}_{x}$ and $\widetilde{\gamma}_{x}$ are sections defined on $W(x)$, one can write

$$
\widetilde{\omega}_{x}(t)=\widetilde{\gamma}_{x}(t) g_{x}(t), \quad g_{x}: W_{\text {loc }}(x) \rightarrow G,
$$

where the functions $g_{x}$ are continuous, vary continuously with $x$, and $g_{x}(x)=\mathrm{Id}$. Use this in (4.7), together with the the same formula for $\widetilde{\gamma}_{x}$ and the $G$-equivariance of $F$, to obtain

$$
g_{x}(t)=\beta(n, x)^{-1} g_{x_{n}}\left(t_{n}\right) \beta(n, x) .
$$

Since $\lim _{n \rightarrow \infty} \operatorname{dist}_{M}\left(t_{n}, x_{n}\right)=0$ and $g_{x}(t)$ is continuous in both $x$ and $t$, we conclude from the biinvariance of the distance on $G$ that

$$
\lim _{n \rightarrow \infty} \operatorname{dist}_{G}\left(\beta(n, x)^{-1} g_{x_{n}}\left(t_{n}\right) \beta(n, x), \text { Id }\right)=\lim _{n \rightarrow \infty} \operatorname{dist}_{G}\left(g_{x_{n}}\left(t_{n}\right), \text { Id }\right)=0,
$$

hence $g_{x} \equiv \operatorname{Id}$ on $W_{\text {loc }}(x)$, which shows that $\widetilde{\gamma}_{x}=\widetilde{\omega}_{x}$, as claimed.

\section{Proof of Lemma 4.3.}

Notice first that since $\widetilde{a}$ commutes with $\widetilde{b}$, the stable foliation of $\widetilde{b}$, defined by (4.1), is preserved by $\widetilde{a}$. Therefore, the intersection $\widetilde{W}:=W_{\widetilde{a}}^{s} \cap W_{\widetilde{b}}^{s}$ is preserved by both $\widetilde{a}$ and $\widetilde{b}$. But $\widetilde{W}$ is a lift of the foliation $W(x):=W^{s}(x ; \alpha(a)) \cap W^{s}(x ; \alpha(b))$. By the uniqueness property of Lemma 4.2, $\widetilde{W}$ is described by the restriction of either $\widetilde{\gamma}^{\widetilde{a}}$ or $\widetilde{\gamma}^{\widetilde{b}}$ to $W$, which is exactly what condition (4.2) states (once the sections are adjusted by the proper element of $G$ so that they intersect).

\section{Foliations in the Smooth CASE}

We prove here the equivalent of Proposition 4.1 for the case of smoothly TNS actions.

Proposition 5.1. Assume that $\mathcal{A} \subset \operatorname{Diff}\left(\mathbb{T}^{n}\right)$ is a smoothly $T N S$ action, $G$ is a compact Lie group, and $P \rightarrow M$ is a principal $G$-bundle that admits a smooth $G$-extension $\widetilde{\alpha}: \mathcal{A} \times P \rightarrow P$ of $\alpha$.

Then there exists a $\widetilde{\alpha}$-invariant smooth horizontal foliation $\widetilde{W}$ of $P$. Moreover, this is the unique continuous $\widetilde{\alpha}$-invariant horizontal foliation of $P$. 
Proof. The proof is along the lines of the proof of Theorem 2.3 in [10]. By [10, Remark $(3), \S 2]$ we can assume that the smooth distributions $E_{i}$ are integrable. Denote by $W_{i}$ the corresponding smooth foliations of $M$. By Lemma 4.2 there are foliations $\widetilde{W}_{i}$ covering $W_{i}$ with smooth leaves which vary continuously in the $C^{\infty}$-topology. Let $\widetilde{E}_{i}$ be the distributions they determine in $P$. We construct the foliation $\widetilde{W}$ as the span of $\widetilde{W^{s}(a)}$ and $\widetilde{W^{u}(a)}$. Using distributions, this translates into showing that the distribution $\mathcal{D}:=\widetilde{E^{s}(a)}+\widetilde{E^{u}(a)}=\sum_{i} \widetilde{E_{i}}$ is integrable. In order to prove this, by the theorem of Frobenius, we have to check that $\mathcal{D}$ is smooth and involutive (i.e. if $X$ and $Y$ are two smooth enough vector fields in $\mathcal{D}$, then $[X, Y] \in \mathcal{D})$.

Let $m=\operatorname{dim} M$ and $l=\operatorname{dim} G$. Since $G$ is parallelizable, one can choose a smooth frame $\left\{Z_{k}\right\}_{k=1, l}$ of $T G$. Let $U \subset M$ be a small open set, and choose a smooth frame $\left\{X_{j}\right\}_{j=1, m}$ of $T M$ over $U$ such that each field $X_{j}$ is contained in some $E_{i}$. In view of the construction of $\mathcal{D}$, one can find a unique frame $\left\{Y_{j}\right\}_{j=1, m}$ over $U \times G$ which spans $\mathcal{D}$ and has the form $Y_{j}=X_{j}+\sum_{k} \beta_{j, k} Z_{k}$, with $\beta_{j, k}: U \times G \rightarrow \mathbb{R}$.

Clearly the functions $\beta_{j, k}$ are smooth in the $G$-variable. To show that $\beta_{j, k}$ is smooth along $E_{i}$, choose an element $c \in S$ for which $X_{j}, E_{i} \subset E^{s}(c)$. Since $Y_{j} \in \widetilde{E^{s}(c)} \subset \mathcal{D}$, the conclusion follows from the fact that $\widetilde{W^{s}(c)}$ has smooth leaves (by Lemma 4.2).

As in [10, Lemma 3.3], this implies that $\mathcal{D}$ is smooth. To complete the proof, let $c \in S$ be such that $X_{i},\left.X_{j} \in E^{s}(c)\right|_{U}$. Then $Y_{i}, Y_{j} \in \widetilde{E^{s}(c)}$, which is involutive (being tangent to a foliation), hence $\left[Y_{i}, Y_{j}\right] \in \widetilde{E^{s}(c)} \subset \mathcal{D}$.

This proves that the foliation $\widetilde{W}$ exists and has smooth leaves.

\section{The Holonomies}

Once we obtain the existence of the horizontal foliation of an extension, we can discuss the classification of these extensions. Again, we parallel the results of [10]. The equivalent of [10, Lemma 4.4] is discussed in $\S 7$, using the Appendix. Lemmas 6.3 and 6.4 replace [10, Lemma 4.5] — we now classify the general case, instead of only small cocycles.

The main purpose of this section is to prove the following:

Theorem 6.1. Let $G$ be a compact connected Lie group, $M$ an infranilmanifold, and $a \in$ $\operatorname{Diff}(M)$ an Anosov diffeomorphism. Then there are only finitely many pairs $(P, \mathcal{F})$, of a principal $G$-bundle $P$ over $M$ and a horizontal foliation $\mathcal{F}$ of $P$, for which there exists a lift of a to a $G$-bundle map $\widetilde{a}: P \rightarrow P$ that preserves $\mathcal{F}$.

Moreover, for each of these choices, the holonomy map of the foliation $\mathcal{F}, H: \pi_{1}(M) \rightarrow G$, has a finite image.

Remark. It is enough to prove the theorem for a power of $a$. Therefore, without loss of generality, we will assume that $a$ has a fixed point, say $x_{0}$. 
We begin by describing the holonomy, and the constraints it has to satisfy if it corresponds to a $G$-extension. Then we prove that under our hypothesis, these constraints admit only finitely many solutions.

Let $p: P \rightarrow M$ be a principal $G$-bundle and $\mathcal{F}$ a continuous $G$-invariant horizontal foliation of $P$. Equivalently, $(P, \mathcal{F})$ describes a principal bundle with group $G^{\text {discrete }}, G$ with the discrete topology.

The pair $(P, \mathcal{F})$ is described by the holonomy $H: \pi_{1}\left(M, x_{0}\right) \rightarrow G$, which is defined as follows. Choose a $G$-equivariant map $\psi: P_{x_{0}} \rightarrow G$, where $P_{x_{0}}:=p^{-1}\left(x_{0}\right)$ (recall that $G$ acts on the right, hence $\psi(\xi g)=\psi(\xi) g)$. Given $\omega \in \pi_{1}\left(M, x_{0}\right)$, take a loop $c:[0,1] \rightarrow M$, $c(0)=c(1)=x_{0}$, that represents $\omega$. Let $\widetilde{c}:[0,1] \rightarrow M$ be a horizontal lift of $c$ (i.e., $\widetilde{c}$ is contained in a leaf of $\mathcal{F})$. Then $\psi(\widetilde{c}(1))=H(\omega) \psi(\widetilde{c}(0))$. Note that by the $G$-invariance of $\mathcal{F}$, any lift of $c$ gives the same value for $H(\omega)$, because they differ by a right translation by an element of $G$.

It is easy to see that $H$ is a homomorphism of groups: $H(\omega \eta)=H(\omega) H(\eta)$, where we use the convention that the paths corresponding to $\omega \eta$ is obtained by first covering $\eta$, and then $\omega$ (hence, the end point of $\eta$ is the starting point $\omega$ ).

Recall that the conjugacy class of $H$ determines the isomorphism class of $(P, \mathcal{F})$ (see $[25$, Theorem 13.9], which we reproduce as Theorem A.2).

For each bundle map $\widetilde{a}: P \rightarrow P$ that preserves the fiber $P_{x_{0}}$, one can introduce $\beta_{\widetilde{a}} \in G$ describing the action of $\widetilde{a}$ in this fiber by

$$
\psi(\widetilde{a}(\xi))=\beta_{\widetilde{a}} \psi(\xi), \quad \xi \in P_{x_{0}},
$$

where $\psi: P_{x_{0}} \rightarrow G$ is the map used in defining the holonomy. Note that $\beta_{\widetilde{a}}$ is independent of $\xi$.

For a group action $\widetilde{\alpha}: \mathcal{A} \times P \rightarrow P$ which preserves $P_{x_{0}}$, this gives a homomorphism $\beta: \mathcal{A} \rightarrow G$.

The following result is a special case of Theorem A.6:

Proposition 6.2. Let $(P, \mathcal{F})$ be a principal bundle with a horizontal foliation, and $\alpha$ : $\mathcal{A} \times M \rightarrow M$ an action on the base that has a fixed point $x_{0}$.

Given a homomorphism $\beta: \mathcal{A} \rightarrow G$, there exists an extension $\widetilde{\alpha}: \mathcal{A} \times P \rightarrow P$ of $\alpha$ that preserves $\mathcal{F}$ and corresponds to $\beta$ if and only if, for each $a \in \mathcal{A}$,

$$
H\left(a_{*} \omega\right)=\beta(a) H(\omega) \beta(a)^{-1}, \quad \omega \in \pi_{1}\left(M, x_{0}\right),
$$

where $a_{*} \in \operatorname{Aut}\left(\pi_{1}\left(M, x_{0}\right)\right)$ is the action induced on the homotopy group by $\alpha(a)$.

We begin now the proof of Theorem 6.1. It consists of two steps. First, we show that under the assumptions of the theorem, the holonomy homomorphism $H$ must have a finite image:

Proposition 6.3. Let $G$ be a compact connected Lie group, $M$ an infranilmanifold, and $a \in \operatorname{Diff}(M)$ an Anosov diffeomorphism which fixes a point $x_{0} \in M$. Pick $g \in G$. Consider the automorphism $a_{*} \in \operatorname{Aut}\left(\pi_{1}\left(M, x_{0}\right)\right)$ induced by a (by the Franks-Manning classification, $a_{*}$ is "hyperbolic"). 
If $H: \pi_{1}\left(M, x_{0}\right) \rightarrow G$ satisfies

$$
H\left(a_{*} \omega\right)=g H(\omega) g^{-1}, \quad \omega \in \pi_{1}(M),
$$

then $H$ has finite image. Moreover, the number of elements in the image of $H$ is bounded by a quantity $N_{\#}(a, G)$ depending only on a and $G$.

Therefore, the map $H: \pi_{1}\left(M, x_{0}\right) \rightarrow G$ is given by a composition of a quotient into a finite group followed by an inclusion, $\pi_{1}\left(M, x_{0}\right) \rightarrow F \hookrightarrow G$, where the order of $F$ is bounded by $N_{\#}(a, G)$. Since $\pi_{1}\left(M, x_{0}\right)$ is finitely generated, there are only finitely many such quotient maps. The next result states the same for the inclusion $F \hookrightarrow G$ :

Proposition 6.4. Let $F$ be a finite group and $G$ a compact connected Lie group. Then, up to conjugacy by an element in $G$, there are at most finitely many homomorphisms $\rho: F \rightarrow G$.

These prove Theorem 6.1. Indeed, let $H$ be the holonomy of the principal bundle $P$. By Proposition 6.2, relation (6.2) holds for some $g \in G$. But then Propositions 6.3 and 6.4 imply that up to conjugacy, there are only finitely many possibilities for $H$. Theorem A.2 concludes the proof.

We now prove the two Propositions stated above.

\section{Proof of Proposition 6.3.}

Since $M$ is an infranilmanifold, $\pi_{1}(M)$ is the extension of a nilpotent group $\Lambda$ by a finite group $F$ :

$$
\{1\} \rightarrow \Lambda \rightarrow \pi_{1}(M) \rightarrow F \rightarrow\{1\},
$$

where $\Lambda$ is a discrete subgroup in a connected, simply connected nilpotent Lie group $N$. The homomorphism $a_{*}$ induced by $a$ on the fundamental group of $M$ is the restriction of an automorphism $\bar{a}$ of $N$ that preserves $\Lambda$. The hyperbolicity assumption means that the differential at the origin of $\bar{a}$ has no eigenvalues on the unit circle (seen as a transformation of the Lie algebra of $N$ ).

Note that it is enough to prove our claim for the restriction of both $H$ and $a_{*}$ to $\Lambda$.

We will proceed by induction on the nilpotency order (i.e., depth) of $N$, using only the properties of $N, \Lambda$ and $\bar{a}$ described above.

Because $N$ is nilpotent, one can identify $N$ and its Lie algebra via the exponential map. In particular, one concludes that all Lie subgroups of $N$ are simply connected. Via this identification, $\bar{a}$ corresponds to its differential at the origin. Recall that all discrete subgroups in a connected nilpotent Lie group are finitely generated (see [21], Corollary 2 to Theorem $2.10)$.

The basic observation is the following:

if $T \subset G$ is an abelian subgroup, then $\mathcal{N}_{G}(T) / \mathcal{Z}_{G}(T)$ is finite and its order is bounded by a constant $N_{\#}(G)$ depending only on $G$,

where $\mathcal{N}_{G}(T):=\left\{g \in G \mid g T g^{-1}=T\right\}$ and $\mathcal{Z}_{G}(T):=\left\{g \in G \mid g t g^{-1}=t\right.$ for all $\left.t \in T\right\}$ are the normalizer, respectively the centralizer, of $T$ in $G$. Indeed, since $G$ is compact, we 
may assume it is a subgroup of a unitary group and conclude that it is enough to check the statement for $G=\mathrm{U}(q)$. But an abelian subgroup $T \subset \mathrm{U}(q)$ can be diagonalized:

$$
V t V^{*}=\operatorname{diag}\left(\lambda_{i}(t)\right) \text { for each } t \in T,
$$

where $\lambda_{i}: T \rightarrow \mathbb{T}, i=1, \ldots, q$, are multiplicative characters of $T$. A direct computation then shows that $\mathcal{N}_{U(q)}(T) / \mathcal{Z}_{U(q)}(T)$ is isomorphic to a subgroup of the permutations of the diagonal that exchange the "blocks" of characters having the same multiplicity. In particular, it can have at most $q$ ! elements.

Consider first the case when $N$ is abelian, thus $\Lambda \cong \mathbb{Z}^{r}$. Since $g$ normalizes $H(\Lambda)$, the previous observation implies that some power of it, say $g^{p}, p \leq N_{\#}(G)$, actually centralizes this image. Thus, $H\left(a_{*}^{p} \omega\right)=H(\omega), \omega \in \Lambda$, which shows that $H$ is trivial on the image $\left(a_{*}^{p}-\operatorname{Id}\right)(\Lambda)$. Since $a_{*}^{p}-\operatorname{Id}$ is invertible on $\Lambda \otimes \mathbb{Q}$, this image is of finite index in $\Lambda$. This proves our claim for $N$ abelian.

Assume now that the assertion was proven for groups $N$ whose nilpotency order is at most $\ell$. If $N$ has nilpotency order $\ell+1$, let $N_{1}:=[N, N]$ and $\Lambda_{1}:=N_{1} \cap \Lambda$, which is a normal subgroup of $\Lambda$ containing $[\Lambda, \Lambda]$. The induction hypothesis applied to $\Lambda_{1} \subset N_{1}$ implies that $H\left(\Lambda_{1}\right) \subset G$ is finite and of bounded order. Thus the normal subgroup $K:=\operatorname{ker}(H) \cap \Lambda_{1}$ of $\Lambda$ has finite index in $\Lambda_{1}$, and therefore $[\Lambda / K, \Lambda / K] \subset \Lambda_{1} / K$ has bounded finite order.

Consider the short exact sequence of groups

$$
\{1\} \rightarrow[\Lambda / K, \Lambda / K] \rightarrow \Lambda / K \rightarrow A \rightarrow\{1\}
$$

whose first term is finite of bounded order, the second is nilpotent of depth at most $\ell+1$, and the third is abelian. We can invoke the following result of Dixmier (see [27], Corollary 2.3): if the center $Z$ of a nilpotent group $L$ has exponent $m$ (i.e., $z^{m}=\operatorname{Id}_{L}$ for each $z \in Z$ ) and the depth of $L$ is $\kappa$, then $L$ has exponent at most $m^{\kappa}$. Thus $A$ has finite exponent. However, $\Lambda$ is finitely generated, hence $A$, and therefore $\Lambda / K$, are actually finite. We thus obtain a finite bound on the order of $\Lambda / K$, and therefore on the size of $H(\Lambda)$ as well.

Remarks. 1. If the group $G$ is not compact, (6.1) admits solutions $H$ that have infinite image. For example, assume that the linear action $\alpha$ of $\mathcal{A}$ on $\mathbb{T}^{d}$ is induced from the homomorphism $\lambda: \mathcal{A} \rightarrow \operatorname{SL}(d, \mathbb{Z})$, and thus $\alpha_{*}=\lambda$ after the identification $\pi_{1}\left(M, x_{0}\right) \cong \mathbb{Z}^{d}$, where $x_{0}$ is the origin. Let $G$ be $\operatorname{SL}(d+1, \mathbb{Z})$. Then

$$
H(\omega)=\left(\begin{array}{cc}
1 & 0 \\
\omega & \mathrm{I}_{d}
\end{array}\right), \quad \beta(a)=\left(\begin{array}{cc}
1 & 0 \\
0 & \lambda(a)
\end{array}\right), \quad \omega \in \pi_{1}\left(M, x_{0}\right), \quad a \in \mathcal{A}
$$

satisfy (6.1).

2. If we do not fix the compact Lie group $G$, then the image of the holonomy homomorphism can be almost any finite quotient of $\pi_{1}\left(M, x_{0}\right)$. E.g., for $\pi_{1}\left(M, x_{0}\right)=\mathbb{Z}^{k}$, one can take $\mathbb{Z}^{k} / p \mathbb{Z}^{k}$ for any $p \geq 1$. We describe this next.

Proposition 6.5. Assume that $\alpha$ is an action of a group $\mathcal{A}$ on the compact manifold $M$ having a fixed point $x_{0}$, and $H: \pi_{1}\left(M, x_{0}\right) \rightarrow F$ is a finite quotient of $\pi_{1}\left(M, x_{0}\right)$ whose 
kernel is invariant under the action $\alpha_{*}$ induced by $\alpha$ on $\pi_{1}\left(M, x_{0}\right)$. Then we can embed $F$ in a compact Lie group $G$ and find $\beta: \mathcal{A} \rightarrow G$ so that equation (6.1) holds.

Proof. The condition on the kernel of $H$ implies that we can quotient $\alpha_{*}$ to an action $\overline{\alpha_{*}}: \mathcal{A} \rightarrow \operatorname{Aut}(F)$. Consider the wreath product $\bar{F}:=F \ltimes \operatorname{Aut}(F)$ and let $\beta:=\alpha_{*}: \mathcal{A} \rightarrow$ $\operatorname{Aut}(F) \subset \bar{F}$. Then $(6.1)$ holds. It only remains to embed the finite group $\bar{F}$ into a compact group (e.g., view $\bar{F}$ as a subgroup of a permutation group and realize the latter by unitary matrices) to conclude the proof.

Proof of Proposition 6.4. Let $\operatorname{Rep}(F, G)$ be the space of homomorphisms from $F$ into $G$, endowed with the compact-open topology. Since $\operatorname{Rep}(F, G)$ is compact, the proposition will follow if we show that any two nearby homomorphisms are conjugate.

Indeed, notice first that if two homomorphisms $\rho, \rho^{\prime}: F \rightarrow G$ are close to each other than they have the same kernel, because there are no small subgroups in $G$. Therefore the conclusion follows from the following theorem of Montgomery and Zippin (see [15]):

Theorem 6.6 (Montgomery and Zippin). Let $G$ be a Lie group and $G^{*}$ a compact subgroup of $G$ (in particular $G^{*}$ can be finite). Then there exists in $G$ an open set $O$ containing $G^{*}$ with the property that for each subgroup $G_{0}$ of $G$ lying in $O$ there is an element $g$ of $G$ such that $g^{-1} G_{0} g$ is in $G^{*}$.

\section{The MAIN RESUlt AND ITS PROOF}

Let $\alpha: \mathcal{A} \times M \rightarrow M$ be a TNS action on the infranilmanifold $M$.

We will discuss the general case, when the action need not have fixed points. Such actions exist, see [9].

Let us first explain why $\mathbb{T}^{\kappa}$-extensions can always be realized on a trivial bundle, i.e., as cocycles. Thus, the first part of Theorems 3.1 and 3.2 follows from the second (since the group $G$ is abelian, relation (3.1) gives elements that are in the kernel of $H$ ). Indeed, any homomorphism $H$ from the abelian group $\mathcal{A}=\mathbb{Z}^{k}$ into $\mathbb{T}^{\kappa}$ can be homotopically deformed to the trivial one (deform the image of each generator of $\mathcal{A}$ separately). Therefore, the bundle corresponding to $H$ is (isomorphic to) the trivial principal bundle, $M \times \mathbb{T}^{\kappa}$. For other groups $G$ we were not able to find a result about the isomorphism class of a principal $G$-bundle specified by a given holonomy $H$.

We describe now the general results. We give only the statement for extensions. As explained above, if $G=\mathbb{T}^{\kappa}$ then we have to consider only cocycles, with the corresponding simplifications.

Theorem 7.1. Let $M$ be an infranilmanifold and $\alpha: \mathcal{A} \times M \rightarrow M$ a smoothly-TNS action.

Since the action is abelian and contains hyperbolic elements, there is a point $x_{1} \in M$ which has a finite $\mathcal{A}$-orbit, $\left\{x_{k} \mid k=1, \ldots, p\right\}$. For each $x_{k} \in \mathcal{A} x_{1}$, choose a path $\omega_{x_{k}}$ from $x_{1}$ to $x_{k}$; as $\omega_{x_{1}}$ choose the constant path. 
Let $G$ be a compact connected Lie group.

The continuous cohomology classes of smooth $G$-extensions of $\alpha$ are in one-to-one correspondence with conjugacy classes of pairs of maps $(H, \beta)$, where:

(1) $H: \pi_{1}\left(M, x_{1}\right) \rightarrow G$ is a homomorphism;

(2) $\beta: \mathcal{A} \rightarrow G$ satisfies:

(a) For all $\gamma \in \pi_{1}\left(M, x_{1}\right)$ and $a \in \mathcal{A}$,

$$
H\left(\omega_{a\left(x_{1}\right)}^{-1} a(\gamma) \omega_{a\left(x_{1}\right)}\right)=\beta(a) H(\gamma) \beta(a)^{-1} .
$$

(b) For each $a, b \in \mathcal{A}$,

$$
H\left(\omega_{a b\left(x_{1}\right)}^{-1} a\left(\omega_{b\left(x_{1}\right)}\right) \omega_{a\left(x_{1}\right)}\right) \beta(a b)=\beta(a) \beta(b) .
$$

Up to conjugacy, there are only finitely many homomorphisms $H$, and each has a finite image.

Therefore, each extension has a finite cover which is a trivial $G$-bundle and on which the lifted action of the finite index subgroup $\mathcal{A}_{1}:=\left\{a \in \mathcal{A} \mid a\left(x_{1}\right)=x_{1}\right\}$ is cohomologous to a constant cocycle. This cover can be chosen to be the same for all $G$-extensions of $\alpha$.

Note that if $x_{1}$ is a fixed point of $\alpha$, then these conditions simplify to the requirements that $H$ and $\beta$ be homomorphisms that satisfy relation (3.1). Otherwise, $\beta$ is only a "twisted homomorphism".

The similar result for Hölder cocycles on a torus is:

Theorem 7.2. Assume that $M \cong \mathbb{T}^{d}, \alpha: \mathcal{A} \times M \rightarrow M$ is a TNS action, and $x_{1} \in M$ has a finite $\mathcal{A}$-orbit. Then the conclusions of Theorem 7.1 hold for continuous cohomology classes of $\theta$-Hölder $G$-extensions.

In particular, any Hölder extension is Hölder cohomologous to a smooth extension.

Remark. The properties (7.1) and (7.2) are the conditions of Theorem A.6, rewritten in (A.8) and (A.9). They can be solved in part (see Theorem A.7). We describe this below. As above, denote by $\mathcal{A}_{1}$ the isotropy group of $x_{1}, \mathcal{A}_{1}:=\left\{a \in \mathcal{A} \mid a\left(x_{1}\right)=x_{1}\right\}$, which has index $p$ in $\mathcal{A}$. Choose a set of representatives $b_{i}$ for $\mathcal{A} / \mathcal{A}_{1}, i=1, \ldots, p$. Denote $\overline{\mathcal{A}_{1}}=\mathcal{A}_{1} \cup\left\{b_{i} \mid i=1, \ldots, p\right\}$.

Then the cohomology classes of $G$-extensions of $\alpha$ are in one-to-one correspondence with conjugacy classes of maps $(H, \beta)$, where:

(1) $H: \pi\left(M, x_{1}\right) \rightarrow G$ is a homomorphism;

(2) $\beta: \overline{\mathcal{A}_{1}} \rightarrow G$ satisfies the conditions:

(a) $\beta\left(e_{\mathcal{A}}\right)=e_{G}$;

(b) For each $g \in \overline{\mathcal{A}_{1}}$ and $\gamma \in \pi_{1}\left(M, x_{1}\right)$,

$$
H\left(\omega_{g\left(x_{1}\right)}^{-1} g(\gamma) \omega_{g\left(x_{1}\right)}\right)=\beta(g) H(\gamma) \beta(g)^{-1} ;
$$

(c) For any $g_{1}, g_{2}, g_{3} \in \overline{\mathcal{A}_{1}}, a \in \mathcal{A}_{1}$ such that $g_{1} g_{2}=g_{3} a$,

$$
\beta\left(g_{3}\right) \beta(a) \beta\left(g_{2}\right)^{-1} \beta\left(g_{1}\right)^{-1}=H\left(\omega_{g_{3}\left(x_{1}\right)}^{-1} g_{1}\left(\omega_{g_{2}\left(x_{1}\right)}\right) \omega_{g\left(x_{1}\right)}\right) \text {. }
$$




\section{Proof of Theorems 7.1 and 7.2 .}

Let $\widetilde{\alpha}: \mathcal{A} \times P \rightarrow P$ be a $G$-extension of $\alpha$.

We begin by describing how the invariant $(H, \beta)$ is associated to an extension. There are two cases:

(1) $M$ is a torus and the extension is Hölder;

(2) $M$ is any infranilmanifold, the action is smoothly TNS, and the extension is smooth.

In the first case we use Proposition 4.1, in the second Proposition 5.1. We conclude that there is a horizontal foliation $\mathcal{F}$ preserved by $\widetilde{\alpha}$. For $(P, \mathcal{F})$ we use the notations introduced in the Appendix $\S \mathrm{A}$.

Fix a $G$-equivariant map $\phi_{x_{1}}: P_{x_{1}} \rightarrow G$. Then there is a unique choice of trivializations $\phi_{x_{k}}: P_{x_{k}} \rightarrow G$ such that the parallel transport along $\omega_{k}$ induced by $\mathcal{F}$ takes $\phi_{x_{1}}^{-1}\left(e_{G}\right)$ to $\phi_{x_{k}}^{-1}\left(e_{G}\right)$ (in the notation of $\left.\S \mathrm{A}, K\left(\omega_{x_{k}}\right)=e_{G}\right)$.

Then $H$ is the holonomy of $\mathcal{F}$ computed at $x_{1}$ with respect to $\phi_{x_{1}}$, whereas $\beta$ is defined by the map

$$
a \in \mathcal{A} \mapsto \beta(a):=\left[\phi_{a\left(x_{1}\right)}(\widetilde{a}(\xi))\right]\left[\phi_{x_{1}}(\xi)\right]^{-1} \in G,
$$

which is independent of $\xi \in P_{x_{1}}$. Here $\widetilde{a}$ stands for $\widetilde{\alpha}(a)$.

Alternatively, $\beta(a)$ is defined as follows: for $\xi \in P_{x_{1}}$, let $\eta \in P_{x_{1}}$ be the parallel transport of $\widetilde{a}(\xi)$ along $\omega_{a\left(x_{1}\right)}^{-1}$. Then

$$
\beta(a):=\left[\phi_{x_{1}}(\eta)\right]\left[\phi_{x_{1}}(\xi)\right]^{-1} \in G .
$$

Theorem A.6 shows that the pair $(H, \beta)$ satisfies the conditions (7.1) and (7.2) (see (A.8) and (A.9); by our choice of the trivializations, $\left.K\left(\omega_{x_{k}}\right)=e_{G}\right)$.

Note that a different choice of $\phi_{x_{1}}$ will yield a conjugate pair $(H, \beta)$ for the invariant (see part 4 of Lemma A.4).

Next, we show that conjugacy classes of $(H, \beta)$ are in one-to-one correspondence with cohomology classes of extensions.

First, we show that if two extensions $\widetilde{\alpha}: \mathcal{A} \times P \rightarrow P$ and $\widetilde{\alpha}^{\prime}: \mathcal{A} \times P^{\prime} \rightarrow P^{\prime}$ are cohomologous, then their invariants are conjugate. Let $F: P \rightarrow P^{\prime}$ be the continuous bundle map for which

$$
\widetilde{\alpha}^{\prime}(a)=F \circ \widetilde{\alpha}(a) \circ F^{-1} \quad \text { for each } a \in \mathcal{A} .
$$

Denote by $\mathcal{F}$ and $\mathcal{F}^{\prime}$ the invariant horizontal foliations of $\widetilde{\alpha}$ and $\widetilde{\alpha}^{\prime}$. Then $F(\mathcal{F})$ is a continuous invariant horizontal foliation for $\widetilde{\alpha}^{\prime}$, hence, by Propositions 4.1 and 5.1, it has to coincide with $\mathcal{F}^{\prime}$. Choose a trivialization $\phi_{x_{1}}$ of $P_{x_{1}}$ and let $\phi_{x_{1}}^{\prime}:=\phi_{x_{1}} \circ F^{-1}$ be the trivialization of $P_{x_{1}}^{\prime}$. Then, measured with respect to these trivializations, the invariants of $\widetilde{\alpha}$ and $\widetilde{\alpha}^{\prime}$ coincide (e.g., use the description (7.3) for $\beta=\beta^{\prime}$ ).

Conversely, assume that the invariants of two extensions $\widetilde{\alpha}: \mathcal{A} \times P \rightarrow P$ and $\widetilde{\alpha}^{\prime}: \mathcal{A} \times P^{\prime} \rightarrow$ $P^{\prime}$ are conjugate. Denote by $\mathcal{F}$, respectively $\mathcal{F}^{\prime}$, the invariant horizontal foliations of $\widetilde{\alpha}$ and $\widetilde{\alpha}^{\prime}$. Because the holonomy parts of the invariants are conjugate, Theorem A.2 implies that there is a (smooth) bundle map $F: P \rightarrow P^{\prime}$ such that $F(\mathcal{F})=\mathcal{F}^{\prime}$. Then the action $\widetilde{\alpha}^{\prime \prime}(a):=F^{-1} \circ \widetilde{\alpha}^{\prime}(a) \circ F, a \in A$, on $P$ preserves $\mathcal{F}$. Fix a trivialization of $P_{x_{1}}$; with respect 
to it, let the invariants of $\widetilde{\alpha}$, respectively $\widetilde{\alpha}^{\prime \prime}$, be $(H, \beta)$ and $\left(H^{\prime \prime}, \beta^{\prime \prime}\right)$. In view of the previous arguments, $(H, \beta)$ and $\left(H^{\prime \prime}, \beta^{\prime \prime}\right)$ are conjugate as well: there is an element $g \in G$ such that $H^{\prime \prime}=\operatorname{Int}_{g} \circ H$ and $\beta^{\prime \prime}=\operatorname{Int}_{g} \circ \beta$. Since both $\widetilde{\alpha}$ and $\widetilde{\alpha}^{\prime \prime}$ have the same invariant horizontal foliation, it follows that $H=H^{\prime \prime}$, hence $g$ commutes with the image of $H$ in $G$. But then the second part of Lemma A.5 implies that there is a bundle map $F_{0}: P \rightarrow P$ that covers the identify, preserves $\mathcal{F}$, and has $\beta_{F_{0}}\left(\operatorname{Id}_{M}\right)=g$. Therefore, the $\beta$-part of the invariants of $\widetilde{\alpha}^{\prime \prime}$ and $F_{0} \circ \widetilde{\alpha} \circ F_{0}$ coincide, which implies that the two actions on $P$ are equal (since $M$ is connected). Hence

$$
F^{-1} \circ \widetilde{\alpha}^{\prime}(a) \circ F=F_{0} \circ \widetilde{\alpha}(a) \circ F_{0}^{-1}, \quad a \in \mathcal{A},
$$

which shows that $\widetilde{\alpha}^{\prime}$ and $\widetilde{\alpha}$ are cohomologous, as desired.

This proves the one-to-one correspondence between cohomology classes of extension and conjugacy classes of pairs $(H, \beta)$.

It remains to check the final statements of the theorems. Apply the Theorem 6.1 to a particular fixed Anosov element of $\mathcal{A}$. We conclude that, up to conjugacy, there are only finitely many possible holonomy maps $H: \pi_{1}\left(M, x_{1}\right) \rightarrow G$, and each has finite image. Denote by $\Gamma_{0} \subset \pi_{1}\left(M, x_{1}\right)$ the intersection of their kernels. Then $\Gamma_{0}$ has finite index in $\pi_{1}\left(M, x_{1}\right)$ (being the kernel of the direct sum of the possible holonomy maps). Since the action of $A_{1}$ has a fixed point, it is topologically conjugated to the abelian linear action on $M$ given by $\mathcal{A}_{1}$ acting on $\pi\left(M, x_{1}\right)$. But $M \cong N / \Gamma$, with $\Gamma \cong \pi_{1}\left(M, x_{0}\right)$ (see the definition of infranilmanifolds in $\S 2$ ). Hence $M_{0}=N / \Gamma_{0}$ is a finite cover of $M$, to which $\left.\alpha\right|_{\mathcal{A}_{1}}$ can be lifted. If $\widetilde{\alpha}: \mathcal{A} \times P \rightarrow P$ is an extension of $\alpha$ described by $(H, \beta)$, then the pull-back $\left(P_{0}, \mathcal{F}_{0}\right)$ of $(P, \mathcal{F})$ to $M_{0}$ has trivial holonomy, and the action $\widetilde{\alpha}$ lifts to the constant cocycle given by $\left.\beta\right|_{\mathcal{A}_{1}}$ on $P_{0}$ (note that $\left.\beta\right|_{\mathcal{A}_{1}}$ is a group homomorphism, and the relations (7.1) and (7.2) are satisfied on $\left.\left(P_{0}, \mathcal{F}_{0}\right)\right)$.

\section{Appendix A. Lifting group aCtions}

Let $X$ be a (smooth) connected manifold. Consider a principal $G$-bundle $P \rightarrow X$ over $X$. Assume that $P$ has a horizontal foliation $\mathcal{F}$. In this section we describe the lifts of an action $\alpha: \mathcal{A} \times X \rightarrow X$ on the base to an action on $P$ which preserves the foliation $\mathcal{F}$.

We were not able to find in the literature these results, and therefore we give more details. Although many of the results state equivalent conditions, in most cases we will discuss only one of the implications, since the other one is obtained in a similar fashion.

Notations. Let $P$ be a $G$-bundle over $X$, and $\mathcal{F}$ a horizontal foliation of $P$.

1. Denote by $\Omega$ the set of paths in $X$. There are two maps $s, t: \Omega \rightarrow X$ which give the source and target (i.e., initial and final point) of a path. For paths that start and end at $x \in X$, we use the notation $\Omega_{x, x}$.

For $a: X \rightarrow X$, denote by $\gamma \mapsto a(\gamma)$ its action on paths $\gamma \in \Omega$.

2. We denote by $P_{x}$ the fiber of $P$ above $x \in X$. For each $x \in X$, we choose a right $G$-equivariant map $\phi_{x}: P_{x} \rightarrow G$ (i.e., $\left.\phi_{x}(\xi g)=\phi_{x}(\xi) g\right)$. 
3. Each $\gamma \in \Omega$ determines a parallel transport $\gamma: P_{s(\gamma)} \rightarrow P_{t(\gamma)}$. Namely, $\gamma(\xi)=\eta$ if $\eta$ is the end point of the horizontal lift of $\gamma$ starting from $\xi \in P_{s(\gamma)}$.

Definition A.1. For $\gamma \in \Omega$, denote by $K(\gamma)$ the holonomy of $\gamma$ :

$$
K(\gamma)=\left[\phi_{t(\gamma)}(\gamma(\xi))\right]\left[\phi_{s(\gamma)}(\xi)\right]^{-1},
$$

which is independent of $\xi \in P_{s(\gamma)}$.

Remark. Since $\mathcal{F}$ is a foliation, $K(\gamma)$ depends only on the homotopy class with fixed end points of $\gamma$. In particular, $K: \Omega_{x_{1}, x_{1}} \rightarrow G$ is exactly the holonomy of the foliation, $H: \pi_{1}\left(X, x_{1}\right) \rightarrow G$.

We recall the basic classification theorem for principal bundles endowed with an invariant horizontal foliation (equivalently, having a discrete structure group, or - in the smooth case - a flat connection).

Theorem A.2 ([25, Theorem 13.9]). Fix $x_{1} \in X$. Two continuous principal G-bundles over $X$ with a continuous horizontal foliation, $\left(P_{1}, \mathcal{F}_{1}\right)$ and $\left(P_{2}, \mathcal{F}_{2}\right)$, are isomorphic (i.e., there exists a bundle isomorphism $F: P_{1} \rightarrow P_{2}$ covering the identity map on $M$, and such that $\left.F\left(\mathcal{F}_{1}\right)=\mathcal{F}_{2}\right)$ if and only if the holonomies

$$
H_{1}, H_{2}: \pi_{1}\left(X, x_{1}\right) \rightarrow G
$$

are conjugated in $G$ : there exists an element $g \in G$ such that $H_{1}(\omega)=g H_{2}(\omega) g^{-1}$ for all $\omega \in \pi_{1}\left(X, x_{1}\right)$.

Definition A.3. For a $G$-bundle map $\widetilde{a}: P \rightarrow P$ which covers the map $a: X \rightarrow X$, define $\beta(a, \cdot): X \rightarrow G$ by

which is independent of $\xi \in P_{x}$.

$$
\beta(a, x)=\left[\phi_{a(x)}(\widetilde{a}(\xi))\right]\left[\phi_{x}(\xi)\right]^{-1},
$$

It would be more precise to write $\beta_{\tilde{a}}$ instead of $\beta$. We will do it only if there is the possibility of confusion. Note that any such map determines uniquely a $G$-map $\widetilde{a}$ (which might not be continuous) that covers $a$.

We summarize a few simple properties.

Lemma A.4. (1) For $\gamma, \omega \in \Omega$,

$$
K(\omega \gamma)=K(\omega) K(\gamma), \quad \text { provided } \quad t(\gamma)=s(\omega)
$$

(we use the convention that $\omega \gamma$ is the path obtained by covering first $\gamma$, then $\omega$ ).

(2) If $\widetilde{a}, \widetilde{b}: P \rightarrow P$ are lifts of $a, b: X \rightarrow X$, then

$$
\beta_{\widetilde{a} \tilde{b}}(a b, x)=\beta_{\widetilde{a}}(a, b x) \beta_{\tilde{b}}(b, x) .
$$

(3) If $\gamma \in \Omega$ starts at $x$ and ends at $a(x) \neq x$, then

$$
K(\gamma)^{-1} \beta_{\widetilde{a}}(a, x) \in G
$$

is independent of the choice of $\phi_{a(x)}$. 
(4) If $\phi_{x}^{\prime}(\xi)=g \phi_{x}(\xi)$ is another trivialization of $P_{x}$, then the value $h=K(\gamma)^{-1} \beta_{\widetilde{a}}(a, x)$ considered in part 3 changes to $\mathrm{ghg}^{-1}$ when computed with respect to $\phi_{x}^{\prime}$. The effect is the same on $K(\gamma), \gamma \in \Omega_{x, x}$.

Proof. The first two properties are immediate. For the third one, note that if $\phi_{a(x)}^{\prime}$ is another trivialization, then there is $g \in G$ such that $\phi_{a(x)}^{\prime}(\xi)=g \phi_{a(x)}(\xi)$. The conclusion follows from the definitions of $K$ and $\beta$. The last part also follows from the definitions.

Lemma A.5. Let $(P, \mathcal{F})$ be as above.

(1) The lift $\widetilde{a}: P \rightarrow P$ of $a: X \rightarrow X$ preserves $\mathcal{F}$ if and only if

$$
K(a(\gamma)) \beta(a, s(\gamma))=\beta(a, t(\gamma)) K(\gamma), \quad \text { for each } \quad \gamma \in \Omega .
$$

(2) Given $x_{1} \in X$, there is lift $\widetilde{a}: P \rightarrow P$ of $a: X \rightarrow X$ that preserves $\mathcal{F}$ with a given $\beta\left(a, x_{1}\right) \in G$ if and only if

$$
K(a(\gamma)) \beta\left(a, x_{1}\right)=\beta\left(a, x_{1}\right) K(\gamma), \quad \text { for each } \quad \gamma \in \Omega_{x_{1}, x_{1}} .
$$

Note that if such a lift exists, it is unique, because $X$ is connected.

Proof. We prove the "only if" implication of the first part. $\widetilde{a}$ preserves $\mathcal{F}$ if and only if, given $\xi, \eta$ in the same leaf of $\mathcal{F}, \widetilde{a}(\xi)$ and $\widetilde{a}(\eta)$ are also in the same leaf. Connect $\xi$ to $\eta$ by a horizontal curve, denote by $\gamma$ its projection to $X$. Then $\gamma(\xi)=\eta$, and $a(\gamma) \widetilde{a}(\xi)=\widetilde{a}(\eta)$. The definitions of $K$ and $\beta$ now give (A.3).

To prove the "if" implication of the second part, choose a family of curves $\left\{\omega_{x}\right\}_{x \in X}$ with $s\left(\omega_{x}\right)=x_{1}$ and $t\left(\omega_{x}\right)=x$. As $\omega_{x_{1}}$ choose the constant curve. Define $\beta(a, \cdot)$ by (A.3):

$$
\beta(a, x):=K\left(a\left(\omega_{x}\right)\right) \beta\left(a, x_{1}\right) K\left(\omega_{x}\right)^{-1} .
$$

We have to show that it satisfies (A.3) for any curve $\gamma \in \Omega$. Apply (A.4) for the curve $\omega_{y}^{-1} \gamma \omega_{x} \in \Omega_{x_{1}, x_{1}}$, where $x=s(\gamma), y=t(\gamma)$. The desired relation follows using (A.5) and (A.1).

Theorem A.6. Let $P \rightarrow X$ be a $G$-bundle with a horizontal $G$-invariant foliation $\mathcal{F}$ whose holonomy is $H: \pi_{1}\left(X, x_{1}\right) \rightarrow G$ (the trivialization of $P_{x_{1}}$ is chosen so that $K(\gamma)=H([\gamma])$ for each $\left.\gamma \in \Omega_{x_{1}, x_{1}}\right)$.

Let $\alpha: \mathcal{A} \times X \rightarrow X$ be an action. Then $\alpha$ admits an $\mathcal{F}$-preserving lift $\widetilde{\alpha}: P \rightarrow P$ corresponding to $\beta\left(\cdot, x_{1}\right): \mathcal{A} \rightarrow G$ (see Lemma A.5) if and only if relations (A.6) and (A.7) are satisfied, where:

$$
K(a(\gamma))=\beta\left(a, x_{1}\right) K(\gamma) \beta\left(a, x_{1}\right)^{-1} \quad \text { for all } \quad \gamma \in \Omega_{x_{1}, x_{1}}, a \in \mathcal{A}
$$

and

$$
\beta\left(a b, x_{1}\right)=\beta\left(a, b x_{1}\right) \beta\left(b, x_{1}\right) \quad \text { for each } \quad a, b \in \mathcal{A} .
$$


Remarks. 1. We assume that $\mathcal{A}$ has the discrete topology, hence we do not need to consider continuity in the $\mathcal{A}$-variable. The continuity in the $X$-variable follows from that of $(P, \mathcal{F})$.

2. One can rewrite conditions (A.6) and (A.7) in a form that involves only curves in $\Omega_{x_{1}, x_{1}}$, and quantities that are independent of the choice of trivializations $\phi_{x}, x \neq x_{1}$ (see part 3 of Lemma A.4). Recall that $\phi_{x_{1}}$ was imposed by the identification of $K$ with $H$.

For example, using (A.1), (A.6) is equivalent to

$$
\begin{aligned}
K\left(\omega_{a\left(x_{1}\right)}^{-1} a(\gamma) \omega_{a\left(x_{1}\right)}\right)=\left[K\left(\omega_{a\left(x_{1}\right)}\right)^{-1} \beta\left(a, x_{1}\right)\right] K(\gamma)\left[K\left(\omega_{a\left(x_{1}\right)}\right)^{-1} \beta\left(a, x_{1}\right)\right]^{-1} \\
\text { for all } \gamma \in \Omega_{x_{1}, x_{1}}, a \in \mathcal{A}
\end{aligned}
$$

and, using (A.5) and (A.1), (A.7) is equivalent to

$$
\begin{aligned}
& K\left(\omega_{a b\left(x_{1}\right)}^{-1} a\left(\omega_{b\left(x_{1}\right)}\right) \omega_{a\left(x_{1}\right)}\right)\left[K\left(\omega_{a b\left(x_{1}\right)}\right)^{-1} \beta\left(a b, x_{1}\right)\right]= \\
& =\left[K\left(\omega_{a\left(x_{1}\right)}\right)^{-1} \beta\left(a, x_{1}\right)\right]\left[K\left(\omega_{b\left(x_{1}\right)}\right)^{-1} \beta\left(b, x_{1}\right)\right] \quad \text { for each } \quad a, b \in \mathcal{A}
\end{aligned}
$$

Proof. We prove only the "if" implication.

By Lemma A.5, (A.6) implies that each $a \in \mathcal{A}$ has a unique $\mathcal{F}$-preserving lift $\widetilde{a}$ to $P$ determined by $\beta\left(a, x_{1}\right)$.

Condition (A.7) means that $\tilde{a} \widetilde{b}(\xi)=\widetilde{(a b)}(\xi)$ for any $\xi \in P_{x_{1}}$. Since $X$ is connected, this implies that $\widetilde{a} \widetilde{b}=\widetilde{(a b)}$ on $P$.

Alternatively, an algebraic proof is obtained as follows: by part 2 of Lemma A.4, we have to show that $\beta(a b, x)=\beta(a, b x) \beta(b, x)$ holds for each $a, b \in \mathcal{A}, x \in X$. But, by (A.3),

$$
\begin{gathered}
\beta\left(a, b x_{1}\right)=K\left(a\left(b\left(\omega_{x}\right)\right)\right) \beta\left(a, b x_{1}\right) K\left(b\left(\omega_{x}\right)\right)^{-1}, \\
\beta\left(b, x_{1}\right)=K\left(b\left(\omega_{x}\right)\right) \beta\left(b, x_{1}\right) K\left(\omega_{x}\right)^{-1}, \\
\beta\left(a b, x_{1}\right)=K\left(a b\left(\omega_{x}\right)\right) \beta\left(a b, x_{1}\right) K\left(\omega_{x}\right)^{-1},
\end{gathered}
$$

which give the desired condition.

We will now specialize Theorem A.6 to our set-up, in which the action has a point with finite orbit (although, finiteness is not crucial for the next theorem).

Theorem A.7. Let $\alpha: \mathcal{A} \times X \rightarrow X$ be an action that has a periodic point $x_{1} \in X$, i.e., the $\mathcal{A}$-orbit of $x_{1}$ is finite, say $\mathcal{A} x_{1}=\left\{x_{k} \mid k=1, \ldots, p\right\}$. For each $x_{k} \in \mathcal{A} x_{1}$ choose a path $\omega_{x_{k}}$ from $x_{1}$ to $x_{k}$; as $\omega_{x_{1}}$ choose the constant path.

The isotropy subgroup of $x_{1}, \mathcal{A}_{1}:=\left\{a \in \mathcal{A} \mid a\left(x_{1}\right)=x_{1}\right\}$ has index $p$ in $\mathcal{A}$. Choose a set of representatives $b_{i}$ for $\mathcal{A} / \mathcal{A}_{1}, i=1, \ldots, p$. Denote $\overline{\mathcal{A}_{1}}=\mathcal{A}_{1} \cup\left\{b_{i} \mid i=1, \ldots, p\right\}$.

Let $P \rightarrow X$ be a $G$-bundle having a $G$-invariant horizontal foliation $\mathcal{F}$. Pick a trivialization of $P_{x_{1}}$; this determines the holonomy $H: \pi_{1}\left(X, x_{1}\right) \rightarrow G$ which characterizes $(P, \mathcal{F})$ (see Theorem A.2). 
Then lifts of $\alpha$ to $P$ are in one-to-one correspondence with functions $\beta(\cdot)=\beta\left(\cdot, x_{1}\right)$ : $\overline{\mathcal{A}_{1}} \rightarrow G$ satisfying the following properties (in order to simplify the notation, we see $H$ defined on $\left.\Omega_{x_{1}, x_{1}}\right)$ :

(1) $\beta\left(e_{\mathcal{A}}\right)=e_{G}$;

(2) For each $g \in \overline{\mathcal{A}_{1}}$ and $\gamma \in \pi_{1}\left(X, x_{1}\right)$,

$$
H\left(\omega_{g\left(x_{1}\right)}^{-1} g(\gamma) \omega_{g\left(x_{1}\right)}\right)=\beta(g) H(\gamma) \beta(g)^{-1}
$$

(3) For any $g_{1}, g_{2}, g_{3} \in \overline{\mathcal{A}_{1}}, a \in \mathcal{A}_{1}$ such that $g_{1} g_{2}=g_{3} a$,

$$
\beta\left(g_{3}\right) \beta(a) \beta\left(g_{2}\right)^{-1} \beta\left(g_{1}\right)^{-1}=H\left(\omega_{g_{3}\left(x_{1}\right)}^{-1} g_{1}\left(\omega_{g_{2}\left(x_{1}\right)}\right) \omega_{g\left(x_{1}\right)}\right) .
$$

Remarks. 1. One could just as well use instead of $\mathcal{A}_{1}$ the normal subgroup $\mathcal{A}_{0}:=\{a \in$ $\mathcal{A} \mid a(y)=y$ for all $\left.y \in \mathcal{A} x_{1}\right\}$ of $\mathcal{A}$, and state the above properties for $\mathcal{A}_{0}$ and a set of representatives of $\mathcal{A} / \mathcal{A}_{0}$. Then $g_{1} g_{2}=g_{3} a$ implies that $g_{3}=g_{2}$ if $g_{1} \in \mathcal{A}_{1}$.

2. For $a \in \mathcal{A}_{1}$, relation (A.10) becomes

$$
H(g(\gamma))=\beta(g) H(\gamma) \beta(x)^{-1}
$$

3. Relation (A.11), together with $\beta\left(e_{\mathcal{A}}\right)=e_{G}$, imply that $\beta: \mathcal{A}_{1} \rightarrow G$ is a homomorphism (take $g_{3}=e_{\mathcal{A}}, g_{1}, g_{2} \in \mathcal{A}_{1}$ ).

Proof. Choose trivializations of $\phi_{x_{k}}: P_{x_{k}} \rightarrow G$ such that $K\left(\omega_{x_{k}}\right)=e_{G}$. Since $H(\gamma)=K(\gamma)$ for $\gamma \in \Omega_{x_{1}, x_{1}}$, relation (A.10) is exactly (A.8), which is equivalent to (A.6) of Theorem A.6, or (A.4) of Lemma A.5. Therefore, each element of $g \in \overline{\mathcal{A}_{1}}$ admits a lift $\widetilde{g}$ to $(P, \mathcal{F})$ corresponding to $\beta(g)$.

For $g_{1}, g_{2}, g_{3} \in \mathcal{A}, a=e_{\mathcal{A}}$, relation (A.11) is exactly (A.9). Therefore, by Theorem A.6, the restriction of $\beta$ to $\mathcal{A}_{1}$ determines a lift of $\alpha$ restricted to $\mathcal{A}_{1}$. We have to check that the lifts $\widetilde{b}_{i}$ of $b_{i}$ are inducing a lift of the whole group $\mathcal{A}$. But $\mathcal{A}$ is generated by $\overline{\mathcal{A}_{1}}$, and the relations that elements of $\overline{\mathcal{A}_{1}}$ satisfy are exactly of the form $g_{1} g_{2}=g_{3} a, a \in \mathcal{A}_{1}$. Relation (A.11) is the necessary and sufficient condition for $\widetilde{g_{1}} \widetilde{g_{2}}=\widetilde{g_{3}} \widetilde{a}$ to hold. Indeed, by (A.2), the latter identity is equivalent to $\beta\left(g_{1}, g_{2}\left(x_{1}\right)\right) \beta\left(g_{2}, x_{1}\right)=\beta\left(g_{3}, a\left(x_{1}\right)\right) \beta\left(a, x_{1}\right)$; use (A.5) and (A.1), together with the fact that $a\left(x_{1}\right)=x_{1}$, to transform it into (A.11).

\section{REFERENCES}

[1] L. Auslander: Bieberbach's theorems on the space groups and discrete uniform subgroups of Lie groups, Annals of Math. 71 (1960), 579-590.

[2] M. Brin, Y. Pesin: Partially hyperbolic dynamical systems, Izv. Akad. Nauk SSSR 38 (1974), $170-212$.

[3] J. Franks: Anosov diffeomorphisms on tori, Trans. AMS 145 (1969), 117-124.

[4] J. Franks: Anosov diffeomorphisms, Global Analysis (Proc. Sympos. Pure Math., vol. XIV, Berkeley, 1968), Amer. Math. Soc., Providence, R.I. (1970), 61-93.

[5] E. R. Goetze, R. J. Spatzier: On Livšic's theorem, superrigidity, and Anosov actions of semisimple Lie groups, Duke Math. J. 88 (1997), 1-27.

[6] M. Hirsch: Differential topology, Graduate Texts in Mathematics 33 (1976), Springer-Verlag. 
[7] M. Hirsch, C. Pugh, M. Shub: Invariant manifolds, Lecture Notes in Mathematics 583 (1977), SpringerVerlag.

[8] S. Hurder: Rigidity of Anosov actions of higher-rank lattices, Ann. Math. 135 (1992), 361-410.

[9] S. Hurder: Affine Anosov actions, Michigan Mathematical Journal 40 (1993), 561-575.

[10] A. Katok, V. Niţică, A. Török: Nonabelian cohomology of abelian Anosov actions, Erg. Th. \& Dyn. Syst. 20 (2000), 259-288.

[11] A. Katok, R. Spatzier: First cohomology of Anosov actions of higher-rank abelian groups and applications to rigidity, Inst. Hautes Études Sci. Publ. Math.79 (1994), 131-156.

[12] A. Livsic: Homology properties of U systems, Math. Notes 10 (1971), 758-763.

[13] A. Livsic: Cohomology of dynamical systems, Math. U.S.S.R., Izvestia 6 (1972), 1278-1301.

[14] A. Manning: There are no new Anosov diffeomorphisms on tori, Amer. Jour. of Math. 96 (1974), $422-429$.

[15] D. Montgomery, L. Zippin: A theorem on Lie groups, Bull. Amer. Math. Soc. 48 (1942), 448-452.

[16] V. Niţică, A. Török: Regularity of the transfer map for cohomologous cocycles, Erg. Th. \& Dyn. Syst. 18 (1998), 1187-1209.

[17] V. Niţică, A. Török: Cohomology of dynamical systems and rigidity of partially hyperbolic actions of higher-rank lattices, Duke Math. Jour. 79 (1995), 751-810.

[18] V. Niţică, A. Török: Local rigidity of certain partially hyperbolic actions of product type, Erg. Th. \& Dyn. Syst.21 (2001), 1213-1237.

[19] W. Parry The Livšic periodic point theorem for non-abelian cocycles, Erg. Th. \& Dyn. Syst. 19 (1999), 687-701.

[20] G. Prasad, M. S. Raghunathan: Cartan subgroups and lattices in semisimple groups, Ann. Math. 96 (1972), 296-317.

[21] M. S. Raghunathan: Discrete subgroups of Lie groups (1972), Springer-Verlag.

[22] K. Schmidt Remarks on Livšic' theory for nonabelian cocycles, Erg. Th. \& Dyn. Syst. 19 (1999), 703721.

[23] M. Shub: Endomorphisms of compact differentiable manifolds, Amer. J. Math. 91 (1969), 175-199.

[24] S. Smale: Differentiable dynamical systems, Bull. Amer. Math. Soc. 73 (1967), 747-817.

[25] N. Steenrod: The topology of fiber bundles, , Princeton University Press (1951),

[26] A. Tognoli: Sulla classificazione dei fibrati analitici reali, Ann. Scuola Norm. Sup. Pisa 21 (1967), 709-743.

[27] R. B. Warfield: Nilpotent groups, Lecture notes in mathematics 513 (1976), Springer-Verlag.

[28] R. Zimmer: Ergodic theory and semisimple groups (1984), Birkhauser, Boston.

VN: Department of Mathematics, 323 Anderson Hall, West Chester University of Pennsylvania, West Chester, PA 19383, and Institute of Mathematics of the Romanian Academy, P.O. Box 1-764, RO-70700 Bucharest, Romania

E-mail address: vnitica@wcupa.edu

AT: University of Houston, Department of Mathematics, 651 PGH, Houston, TX 772043008, and Institute of Mathematics of the Romanian Academy, P.O. Box 1-764, RO-70700 Bucharest, Romania

E-mail address: torok@math.uh.edu 